\title{
Mechanical spectral signatures of malignant disease? A small-sample, comparative study of continuum vs. nano-biomechanical data analyses
}

\author{
Jun $\mathrm{Liu}^{\mathrm{a}}$ and Mauro Ferrari ${ }^{\mathrm{a}, \mathrm{b}, *}$ \\ ${ }^{\mathrm{a}}$ Biomedical Engineering Center, and ${ }^{\mathrm{b}}$ Department of Internal Medicine, The Ohio State University, Columbus, \\ OH 43210, USA
}

\begin{abstract}
Thin sections from human breast biopsies were employed to perform a differential analysis of the ultrasound spectral responses from invasive ductal carcinoma and normal tissue. A non-destructive testing methodology was employed, yielding the reflection coefficients as function of frequency in the clinical ultrasound range. The spectral responses were simulated both in the context of continuum and nano-biomechanics, with the objective of quantifying the physical properties that determine the differences in the spectral signature of normal vs. malignant tissue. The properties that were employed for the theoretical reconstruction of the spectra were: the density, the continuum and the nanomechanical elastic constants, and the nanomechanical theory internodal distance. The latter is a measure of the depth-of-penetration of mechanical actions between contiguous tissue elements. Together with vectorial descriptors of the tissue spatial arrangement, the internodal distance variable affords the quantitative incorporation of tissue architectural data in the theoretical model.

In this paper, the validity of the nanomechanical approach to tissue characterization is discussed, and its potential extensions to biomolecular marker-based cancer diagnostics and therapeutics are considered.
\end{abstract}

Keywords: Invasive ductal carcinoma, nanomechanics, physical properties of tissue, cancer markers, tissue microstructure, ultrasound, biomechanics

\section{Introduction}

In the search for new and more powerful cancer signatures that will enable effective early detection, definitive diagnosis and strategic treatment design, biomolecular markers are justifiably attracting substantial attention [1]. At the current state, however, there remain significant barriers to the application of molecular biomarkers to cancer screening [2]. For example, biomarkers are usually not conveniently avail-

* Address for correspondence: Mauro Ferrari, Ph.D., Biomedical Engineering Center, The Ohio State University, 1080 Carmack Road, Columbus OH 43210, USA. Tel.: +1 614292 4756; Fax: +1 614 292 7301; E-mail: Ferrari.5@osu.edu. able through non-invasive examination, except through sources such as blood [3], urine [4], or fluids containing exfoliated cells $[5,6]$. Consensus is solidifying behind the identification of three major challenges, to the development of technologies that will allow for the biomarker-regulated management of disease, proliferative and otherwise, in the ambulatory population [7]: 1) The identification of clinically effective biomarkers for broad classes of pathologies; 2) The establishment of biofouling-resistant, long-term implantable biosensing technology; and 3) The development of 'smart' delivery system for biopharmaceuticals and other therapeutic molecules. The problem of the biofouling of biomarkers-sensing surfaces in implants, and the associated rapid loss of function, are especially concerning, 
in view of the fact that, despite promising recent advances [8], the development of permanent, implantable biosensing technologies has proven to be particularly elusive. On these grounds, attention was recommended to the pursuits of 'biofouling-insensitive' new marker sensing technologies [7].

Biomarkers and biochemical information represent but one category of cancer "signatures" that could be utilized for early detection, diagnosis and treatment. Cancer, obviously, manifests itself at scales other than the molecular: Cell morphology and the spatial arrangements of cells and tissue carry information that is routinely used by pathologists to diagnose cancer. Most of the current imaging modalities for noninvasive early detection of cancer are based on information at the tissue and organ scales. Conventional imaging technologies such as X-ray CT or ultrasound utilize the contrast in physical properties such as density or acoustical impedance to produce images that are indicative of suspicious regions. Potential advantages offered by the mapping and imaging of magnetic, thermal, electrical, and optical properties are being actively investigated in the context of early breast cancer diagnostics [9].

Of particular relevance to the approach that is presented in this article is the investigation on tissue mechanical properties, or tissue elasticity $[10,11]$. An example of this type of properties is the stiffness of the tissue, e.g., Young's modulus, which could be several folds higher in malignant deposits than in normal tissue [12]. The underlying hypothesis of these approaches is that malignant diseases could be identified through variations in physical properties at the tissue or organ level, and their influence on the images they generate by way of different modalities. This methodology bypasses the issue of fouling, but suffers the drawbacks of resolutions typically not compatible with the cellular and molecular scales. Furthermore, precise quantitative information on the physical properties of the tested biological domains is difficult to obtain by conventional imaging modalities. Recourse may be made, at best, to gray-scale quantitations that are indicative of differences in agglomerate properties, but these do not offer information on the properties themselves, at molecular or cellular scales.

To clarify the point, reference is made to medical ultrasound imaging, in which variations of a complex combination of tissue density and elastic constants result is observable domain contours. Yet, from these it is not possible to reconstruct the local values of these physical variables, and thus the opportunity for the exploitation of these variables as markers of disease is lost. Quantitative measurements of ultrasound propagation velocities and reflections are however possible, and have indeed been used with great success for the non-destructive testing of engineering structures, and the determination of their material properties [13].

A major problem in the translation of this potentially beneficial approach to biomedicine is the fact that appropriate theoretical models must be applied, to allow for the reconstruction of the appropriate physical properties from measured ultrasound propagation data. In particular, the conventional mechanics of solids, including biological domains, is based on a continuum representation [14], that is one that postulates the existence of a typical dimension or Representative Volume Element (RVE) below which matter may be assumed to be continuous and fully homogeneous. On these foundations, mechanical phenomena may then be conveniently and successfully represented in a differential equation format. This extraordinarily successful modeling strategy, however, breaks down anytime it is not possible to establish a continuum RVE aC which is the case when phenomena are examined, that happen on a length scale at which the discrete, inhomogeneous nature of matter is evident, as frequently happens in biology. Approaches were developed, that attempt to address these concern by representing complex composite domains as continua with continuum inclusions [15]. These theories, which were collectively given the name "micromechanics" in the scientific literature, still suffer from the limitation that they do not incorporate the discrete nature of matter, while remaining computationally manageable at domain sizes that are currently not negotiable with lattice dynamics [16], ab-initio approaches or molecular dynamics [17]. To distinguish from micromechanics, the name "nanomechanics" has been employed to denote an approach [18] that represents matter as a discrete set of points at finite distances, as small as the nanometer range, if necessary - that is, the dimensional range at which the discrete nature of matter may no longer be ignored, no matter what the application.

In this paper, we comparatively employ the methods of continuum mechanics and nanomechanics to reconstruct the physical properties of tissue sections obtained from human breast biopsies. The objectives of the study include proposing a test methodology that may be of aid in the practice of pathology, and contributing to the understanding of the boundaries of applicability of continuum and nano-mechanics in quantitative tissue characterization. Speculative extensions of the described quantitative ultrasound method to minimally 


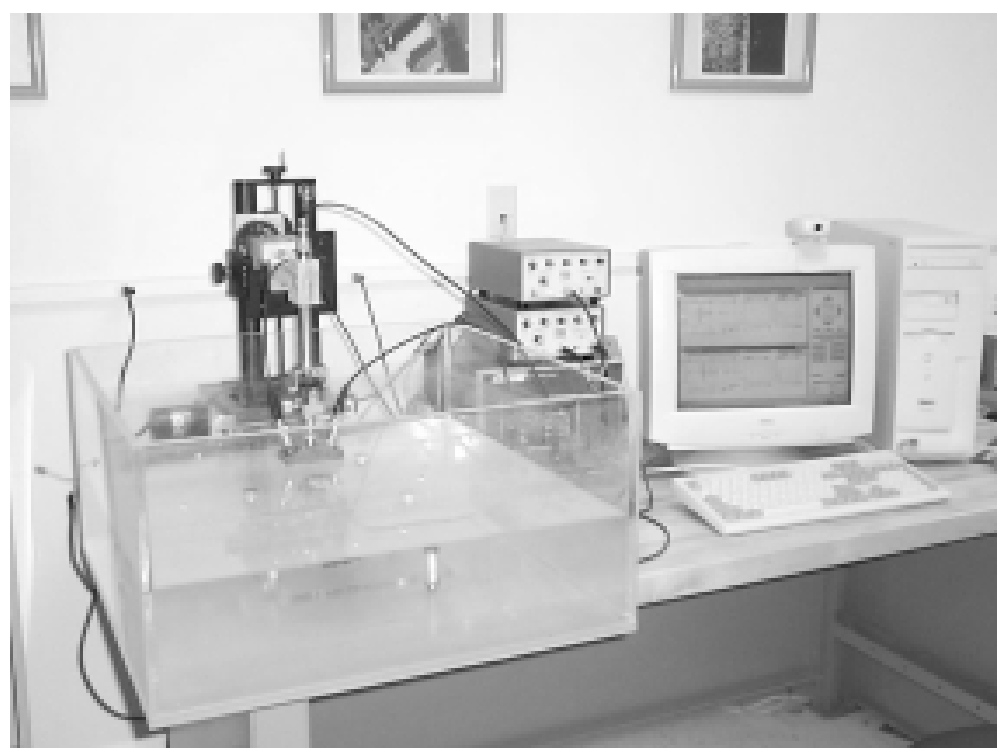

Fig. 1. The ultrasonic non-destructive evaluation system for tissue analysis.

invasive, early-detection and screening practices, are also discussed. The construction of a database for differentiating normal and malignant tissue from quantitative ultrasound testing is under development, but is not the focus of this paper.

\section{Materials and methods}

\subsection{Experimental protocol}

Breast tissue with invasive ductal carcinoma and its normal controls from six patients were procured as frozen blocks through the CHTN (Cooperative Human Tissue Network) at the Ohio State University. The frozen blocks were cut to thin sections (about $150 \mu \mathrm{m}$ ) using a cryostat microtome. The tissue sections were then mounted between two thick glass slides (about $3 \mathrm{~mm}$ ) to make a sandwich structure for the ultrasonic examination. The density of the glass was calculated from the direct measurement of its mass and volume. The longitudinal and shear ultrasonic wave speeds in the glass were measured using standard time-of-flight method [19].

The experimental setup (shown in Fig. 1) was an immersion type non-destructive evaluation system, where the tissue sandwich structures were placed in water during the measurement. The ultrasonic system was composed of three unfocused broadband transducers (Panametrics, central frequency $10 \mathrm{MHz}$, diameter $\frac{1}{2}$ inch), a set of pulser/receiver (Panametrics, 5052PR), and a waveform digitizer (SignaTec, PDA500M, sampling rate $125 \mathrm{MHz}$ ). A computer with a graphic user interface was connected to the ultrasonic system for realtime data acquisition and pre-processing. The reflection spectra were generated from the reflected pulses using a Fast Fourier Transformation (FFT) algorithm that was built into the interface software. The computer also controlled an XYZ translation system (Velmax 86) in which the transducers were mounted.

The vertical and horizontal positions of the transducers were adjusted before each measurement to ensure that the incident ultrasound beams were centered at the tissue plane. Longitudinal plane waves were applied at a $17^{\circ}$ angle with respect to the perpendicular direction against the sample surface. This angle was chosen to maximize the measurement sensitivity [13]. Multiple reflections were collected by a receiver transducer placed at the same angle but the other side of the perpendicular line. Three measurements were performed on each sample to reduce operator error.

\subsection{Mechanical models}

Two theoretical models, one employing nanomechanics, the other based on conventional continuum mechanics, were constructed to comparatively interpret experimental data, and examine the differences between the responses of the normal and malignant tissues. The models were built to simulate the wave propagation and quantitatively correlate the response of the 


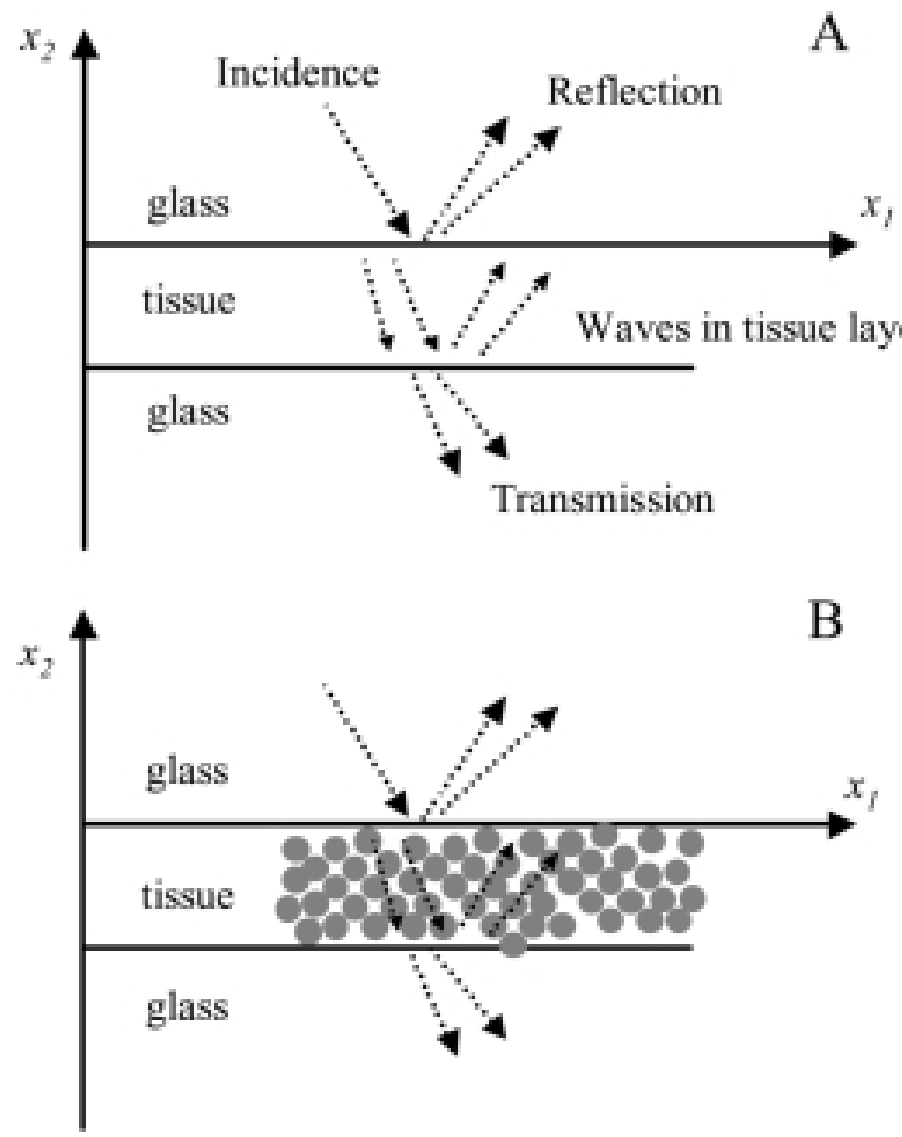

Fig. 2. A: The schematics of waves existing in the sandwich structure upon plan ultrasound incidence; B: Discrete representation of the tissue layer in the nanomechanics model.

tissue samples (the reflection spectra) to the physical properties of the tissue.

The construction of both models is briefly presented herein. Additional information on the nanomechanical analysis may be found in [20].

To construct the theoretical models, displacements were assumed to be in the forms:

$$
\begin{aligned}
\mathbf{u}^{(i)}= & \mathbf{A}_{i} \exp \\
& \left(i k_{i}\left(x_{1} \sin \theta_{i}-x_{2} \cos \theta_{i}-c_{i} t\right)\right)
\end{aligned}
$$

where $\mathbf{u}^{(i)}$ is the displacement of the $i$ th wave, $\mathbf{A}_{i}$ is the amplitude of the displacement, $k_{i}$ is the wave number, $\theta_{i}$ is the propagation angle with respect to the perpendicular direction, and $c_{i}$ is the wave speed.

These forms of the displacements automatically satisfy the respective wave equations in both the continuum and nanomechanical models. Thus, they represent a complete solution, if the appropriate boundary and continuity conditions are met. For the present case, these conditions are derived from the model represented in Fig. 2.

Assuming the mechanical bonds between the glass and the tissue are perfect, the following continuity conditions at each interface are:

1) The continuity of the normal displacement;

2) The continuity of the normal stress;

3) The continuity of the shear displacement;

4) The continuity of the shear stress.

Imposing these conditions, a system of linear equations was obtained, from which the unknown magnitudes of wave displacements $\mathbf{u}^{(i)}$ were solved for.

The reflection/transmission coefficients, which were defined as the ratios of magnitudes of the reflection/ transmission wave over the incident wave, were computed as follows:

$$
R_{s}(f)=M_{R}(f) / M_{I}(f)
$$

where $R_{s}(f)$ is the reflection/transmission coefficient at frequency $f, M_{R}(f)$ is the magnitude of the re- 
flection/transmission at frequency $f$, and $M_{I}(f)$ is the magnitude of the incidence at frequency $f$. A MatLab program was created to give numerical solutions for the reflection coefficients at any frequency, given that the physical properties of the glass were known and those of the tissue were arbitrarily specified.

The reflection spectrum was generated by computing the reflection coefficients for multiple frequencies within a certain range, which was therefore a function of the specific set of physical properties of the tissue.

In the continuum mechanics model, both the layers of the glass and the tissue were assumed to be isotropic and elastic continua. In the nanomechanics model, the thin layer of tissue was represented as discrete nodes (as shown in Fig. 2(B)), while the glass layers remained as continua for simplicity. A spatial arrangement of the nodes was chosen to yield a three dimensional isotropic medium at the macro scale [21]. Within this micro architecture, each node relates to six other nodes at each octant as shown in Fig. 3. Micro level physical properties including the internodal distance $\eta$, the orientation vector $\tau$ and the micro elastic constants $A_{\alpha \beta}$ were specified for each pair of nodes (named as doublets). The reflection spectra in nanomechanics were thus obtained by specifying the micro level physical properties of the tissue layer.

\subsection{Reconstruction algorithm}

An inverse algorithm was applied to reconstruct the quantitative information on each parameter of the tissue properties, for both the continuum- and the nanomechanics based models. These parameters include density, Young's modulus and shear modulus for continuum model; and density, micro elastic constants, and internodal distance, for nanomechanics. A least square minimization method was employed to search for the optimally estimated values on the parameters by solving:

$$
\min _{x_{i} \in R^{n}} \frac{1}{2} \sum_{i=1}^{m}\left(\left|R_{i}^{e}\right|-\left|R_{i}^{s}\right|\right)^{2}
$$

where $x_{i}$ 's are the reconstructed parameters, $n$ is the number of the parameters to be found, $m$ is the number of data points at different frequencies, and $R^{e}$ and $R^{s}$ are the experimental reflection coefficients and simulated reflection coefficients, respectively.

A two-tailed, unequal variance, student T-test was performed on the reconstructed values to test whether there was difference between normal and malignant tissue.

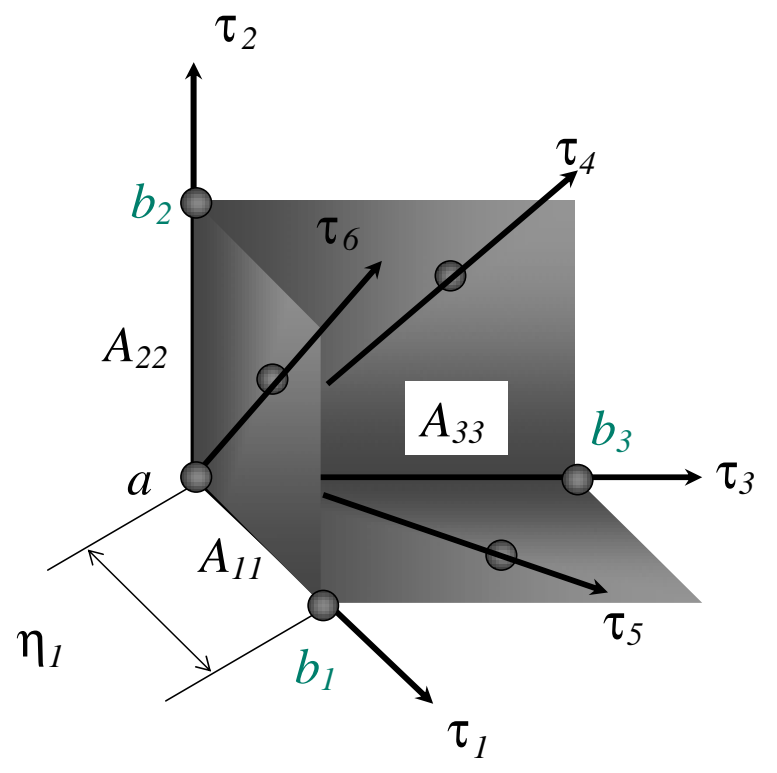

Fig. 3. The spatial arrangement of discrete nodes in the nanomechanics model. Six doublets are shown in the figure: a-b1, a-b2, a-b3, $\mathrm{a}-\mathrm{b} 4, \mathrm{a}-\mathrm{b} 5$ and a-b6. The distance between the nodes is exampled by $\eta_{1}$ for doublet a-b1. In this study, all internodal distances are assumed to be same. The orientations of the nodes are specified by unit vectors $\tau_{1}, \tau_{2}, \tau_{3}, \tau_{4}, \tau_{5}$ and $\tau_{6}$. The micro elastic constants are represented by a matrix of $A_{\alpha \beta}$ with examples of $A_{11}, A_{12}$ and $A_{13}$ shown in the figure.

\section{Results}

With the current settings, both the time domain and the frequency domain reflections were obtained from a few human breast tissue samples. Figure 3 shows the reflection spectra from three malignant breast tissue sections (invasive ductal carcinoma) and the three normal controls from the same patient.

The results of the reconstructed parameters and their statistical analysis are shown in Tables 1 and 2.

\section{Discussion}

Figure 4 shows that the reflection spectra (and, therefore, the mechanical responses) from invasive ductal carcinoma tissue and normal tissue are observably different. For example, the location of the minima, the distance between the two minima, and the depth of the minima are different for the two tissue categories. The very fact that differential mechanical responses are found may suffice for diagnostic and prognostic purposes. However, in order to obtain quantitative information on the physical properties of the tissue, it is nec- 
Table 1

Reconstructed values of the physical properties including density $\rho$, Young's modulus $E$ and shear modulus $\mu$ of tissue from the theoretical framework of continuum mechanics

\begin{tabular}{lcccccc}
\hline & \multicolumn{2}{c}{ Malignant } & & \multicolumn{2}{c}{ Normal } & T Test \\
\cline { 2 - 3 } & Mean $(n=3)$ & SD & & Mean $(n=3)$ & SD & P values \\
\hline$\rho\left(\mathrm{g} / \mathrm{cm}^{3}\right)$ & 0.9796 & 0.0788 & & 0.8728 & 0.0469 & 0.1298 \\
$E(\mathrm{Gpa})$ & 0.1304 & 0.0214 & & 0.1135 & 0.0449 & 0.5975 \\
$\mu(\mathrm{Gpa})$ & 0.0438 & 0.0072 & & 0.0381 & 0.0152 & 0.6046 \\
\hline
\end{tabular}

essary to further interpret the data, employing appropriate theoretical frameworks. To proceed with a differential analysis of continuum and nano-mechanics, and their respective merits in the present context, both approaches are now briefly reviewed, in their essential elements.

In continuum mechanics, the propagation of plane elastic longitudinal and shear waves are governed by the following equations, respectively:

$$
\begin{aligned}
& (\lambda+2 \mu) \frac{\partial^{2} u}{\partial x^{2}}=\rho \frac{\partial^{2} u}{\partial t^{2}} \\
& \mu \frac{\partial^{2} u}{\partial x^{2}}=\rho \frac{\partial^{2} u}{\partial t^{2}}
\end{aligned}
$$

where $x$ is the axis along the direction of wave propagation, $u$ is the displacement, and $\lambda$ and $\mu$ are Lame's elasticity constants. In continuum mechanics, the most general isotropic material has two independent elastic properties. Young' s modulus $E$, can be obtained from $\lambda$ and $\mu$.

The governing equations of the theory of nanomechanics are introduced in its simplest multi-scale variant, which corresponds to the case of node-level, axial only, linear elasticity. More general versions of the theory can be found in [18].

In the linear elastic context, the node-level properties relating axial stress and strain are:

$$
p_{\alpha}=\sum_{\beta} A_{\alpha \beta} \varepsilon_{\beta}
$$

where $p_{\alpha}$ is the overall nodal stress in the $\alpha$-doublet, and $\varepsilon_{\beta}$ is the axial nodal strain associated with $\beta$ doublet, $A_{\alpha \beta}$ is the micromodulus between nodes $\alpha$ and $\beta$. Thus, $A_{\alpha \beta}$ 's are the node-level counterparts of Lame's constants in continuum mechanics.

The strain in the $\alpha$-doublet is computed as follows:

$$
\begin{aligned}
\varepsilon_{\alpha}= & \sum_{i, j=1}^{3} \tau_{\alpha i} \tau_{\alpha j} \frac{\partial u_{i}}{\partial x_{j}} \\
& +\frac{1}{2} \eta_{\alpha} \sum_{i, j, k=1}^{3} \tau_{\alpha i} \tau_{\alpha j} \tau_{\alpha k} \frac{\partial^{2} u_{i}}{\partial x_{j} \partial x_{k}}
\end{aligned}
$$

where $\varepsilon_{\alpha}$ is the nodal strain associated with node $\alpha, \tau$ 's are the direction cosines of the unit vectors connecting two nodes, $u_{1}$ is the displacement at $x_{1}$ direction, $u_{2}$ is the displacement at $x_{2}$ direction, and $\eta_{\alpha}$ is the internal distance associated with node $\alpha$. This distance may be interpreted as the effective radius of penetration of the mechanical contact forcing along the $\alpha$-node direction.

Nanomechanics affords the expression of the macro (continuum) stresses in terms of micro stresses and architecture. This feature is crucial for the analysis of tissue microstructural characteristics based on information measured at the macro-level. The transition relationship is:

$$
\sigma_{i j}=\sum_{\alpha=1}^{n}\left(\tau_{\alpha i} \tau_{\alpha j} p_{\alpha}-\frac{\eta}{2} \tau_{\alpha i} \tau_{\alpha j} \tau_{\alpha k} \frac{\partial p_{\alpha}}{\partial x_{k}}\right)
$$

where $\sigma_{i j}$ is the symmetric, second-rank continuum stress tensor.

The governing equation for the propagation of elastic plane waves also takes a different format compared to that under continuum mechanics. We present here the non-scale wave equation, which is completely equivalent to continuum mechanics, and the presently employed scaling variant:

For scale 1,

$$
\sum_{\alpha=1}^{n} \sum_{\beta=1}^{n} A_{\alpha \beta} \tau_{\alpha i} \tau_{\alpha j} \tau_{\beta k} \tau_{\beta l} \frac{\partial^{2} u_{k}}{\partial x_{l} \partial x_{j}}=\rho \frac{\partial^{2} u_{i}}{\partial t^{2}}
$$

For scale 2,

$$
\begin{aligned}
& \sum_{\alpha=1}^{n} \sum_{\beta=1}^{n} A_{\alpha \beta}\left[\tau_{\alpha i} \tau_{\alpha k_{1}} \tau_{\beta j} \tau_{\beta p_{1}} \frac{\partial^{2} u_{j}}{\partial x_{k_{1}} \partial x_{p_{1}}}\right. \\
& -\frac{\left(\eta_{\alpha}\right)^{2}}{4} \tau_{\alpha i} \tau_{\alpha k_{1}} \tau_{\alpha k_{2}} \tau_{\beta j} \tau_{\beta p_{1}} \tau_{\beta p_{2}} \\
& \left.\frac{\partial^{4} u_{j}}{\partial x_{k_{1}} \partial x_{k_{2}} \partial x_{p_{1}} \partial x_{p_{2}}}\right] \\
= & \rho \frac{\partial^{2} u_{i}}{\partial t^{2}}
\end{aligned}
$$

We are now in position to continue the comparative analysis of the models, in the context of the present 
Table 2

Reconstructed values for micro level physical properties including density $\rho$, micro elastic constants $A_{11}$ and $A_{44}$, and the internodal distance $\eta$ of tissue from the theoretical framework of Nanomechanics

\begin{tabular}{lcccccc}
\hline & \multicolumn{2}{c}{ Malignant } & & \multicolumn{2}{c}{ Normal } & T Test \\
\cline { 2 - 3 } \cline { 5 - 6 } & Mean $(n=3)$ & SD & & Mean $(n=3)$ & SD & P values \\
\hline$\rho\left(\mathrm{g} / \mathrm{cm}^{3}\right)$ & 0.8315 & 0.0233 & & 0.8147 & 0.0589 & 0.6813 \\
$A_{11}(\mathrm{Gpa})$ & 2.1637 & 0.0571 & & 1.7836 & 0.0626 & 0.0015 \\
$A_{44}(\mathrm{Gpa})$ & 0.0523 & 0.0192 & & 0.2202 & 0.0327 & 0.0035 \\
$\eta(\mathrm{mm})$ & 0.0065 & 0.0011 & & 0.0026 & 0.0008 & 0.0091 \\
\hline
\end{tabular}

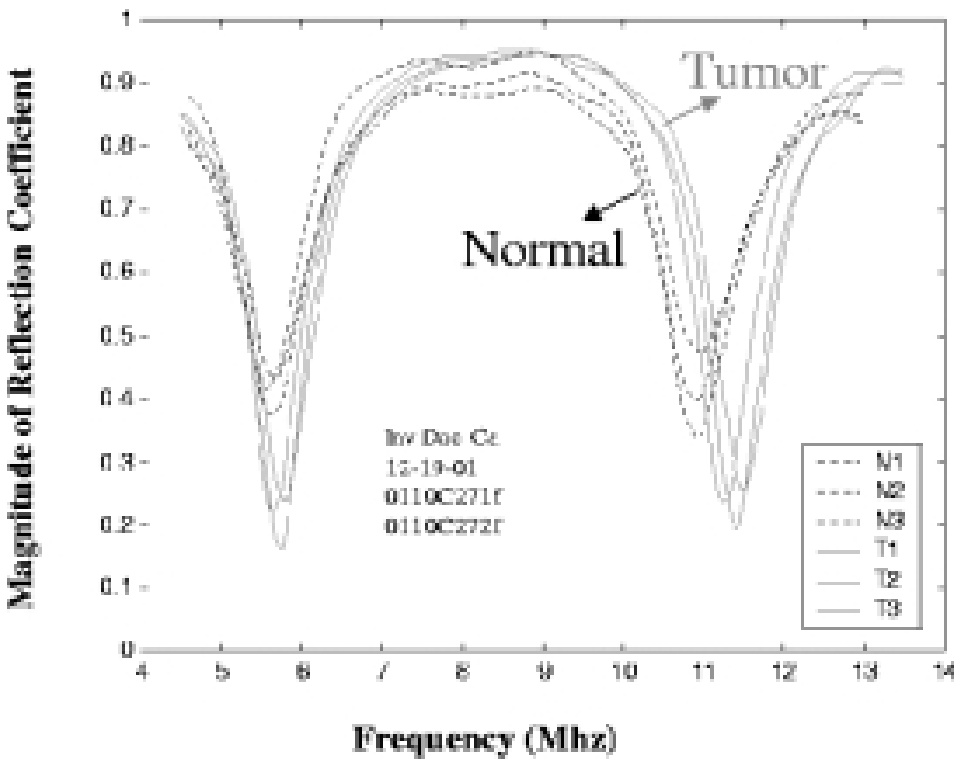

Fig. 4. Experimentally measured reflection spectra from normal breast tissue and malignant breast tissue (invasive ductal carcinoma). N1, N2 and $\mathrm{N} 3$ are the reflection spectra from three normal tissue sections; T1, T2 and T3 are those from three malignant tissue sections. All the tissue sections were obtained from one patient. The frequency range of the ultrasound waves for the measurements were set to 5 to $14 \mathrm{MHz}$.

experiments: The reconstruction shown in Tables 1 and 2 gives the mean values and the standard deviation of several physical properties of a few tissue sections $(n=3)$. In continuum mechanics, the properties are limited, by necessity, to Young's modulus E, the shear modulus $\mu$, and the attenuation coefficients. No information about tissue scaling and architecture may be provided through this continuum model. In the nanomechanical reconstruction, the node-level elastic constants $A_{11}$ and $A_{44}$ and their attenuation counterparts were reconstructed along with the parameters of the micro architecture: the internodal distance $\eta$. The orientation of the nodes (the $\tau$ 's) were defined as in [21] to maintain a three dimensional isotropic arrangement at the macro level, but could potentially left open as parameters to be reconstructed, if so desired.

The reconstructed elasticity values from the continuum model were in general agreement with the literature [12]. However, it was found that the continuum properties of malignant and normal tissues did not differ in a statistically significant fashion $(P>0.05)$. Interestingly, when the same set of experimental measurements was analyzed through the nanomechanical model, the reconstruction yielded parameters that differed in a statistically different manner $(P<0.05)$ between adjacent normal and diseased tissue from the same person. Significant parameters included both node-level elastic constants $A_{11}$ and $A_{44}$, as well as the effective internodal distance $\eta$.

Studies on larger sample sizes will obviously be required, to validate the encouraging indication found in this preliminary study. The creation of a database, which includes different breast malignancies is currently being pursued.

Venturing into the domain of speculations into the future, it may be expected that the nanomechanical analysis approach might be employed in-vivo, for screening or diagnostic purposes. This would require the ap- 
plication of multiple transducers, to obtain the reflection and transmission signals at different locations of an organ. The spatial map of the reconstructed values of certain parameters could be plotted as images. The quantitative information of the parameters at any interested region could be made available simultaneously. Obvious impediments to this potential approach will be concerns about depth-of-penetration of the mechanical signal, tissue damage at the frequencies that allow for deeper penetration, and inhomogeneity-driven effects such as dispersion and attenuation phenomena. These concerns are no longer relevant, if attention is restricted to surface probing only: Transducer arrays are currently being developed within our group [22] that will test the local nanomechanical response of surface tissue, for both screening and diagnostic applications.

Another potential direction for future extension of the proposed approach is the use of biologically targeted micro- or nano-particles as mechanical signal amplifiers, or nanomechanical 'smart contrast agents'. The key notion is that the particles could be conjugated to biologically targeting agents, with pronounced affinity for molecular biomarkers associated with pathologies of interest. The particles could be fabricated so as so maximize the nanomechanical contrast with respect to normal adjacent tissue, for instance by judicious choice of material and geometry. By contrast with current trend in microbubble-enhanced ultrasound imaging, the contrast particles would be fabricated with materials of much greater elastic properties that the biological surroundings. Approaches of this kind may be envisioned for in-vitro applications, as contrast agents for rapid, automated pathology slide readers, but also as enhancers of the biomarker-targeted, quantitative ultrasound probing of skin and internal surfaces, accessible via minimally invasive scopes, and catheters.

Further into the future, it might be investigated whether ultrasound-based systems may be employed as a single-platform technology, that is, a method for performing diagnostics, and administering therapy from the same device or system. Certainly, biomarkertargeting drug-delivery micro- and nano-particles are already under study [23,24]. Also, methods for inducing the rupture of biological and synthetic materials have been available for quite some time, and are routinely employed in clinical settings $[25,26]$. If biomarker-targeted, microparticle-enhanced ultrasound diagnostics and screening are to become a clinical reality, the next step could be the integration of the ultrasound-activated release of therapeutic payload from the particles - thus establishing a complete singleplatform oncological technology.

\section{Conclusion}

Thin sections of invasive ductal carcinoma and normal tissue were obtained from a small number of human breast tissue biopsies. The sections were tested employing a quantitative, ultrasound-based, nondestructive protocol. The reflection coefficients of sections were obtained, as functions of a broad window of frequencies in the clinical ultrasound range. The spectral responses of the normal and malignant sections were found to be remarkably different. In order to obtain quantitative information on the physical variables, which underlie the spectral differences, the spectra were reconstructed employing both the methods of continuum and nano-biomechanics. Continuum-level elastic properties such as the Young's modulus and the shear modulus were found not to differ in a statistically significant fashion, between the normal and malignant tissue. The nanomechanical reconstructions, by contrast, yielded statistical significant differences in nodelevel elastic properties, and the internodal distance, and therefore proposed these quantities as novel potential markers of invasive ductal carcinoma. These results appear to encourage further investigations on the application of nano-biomechanics to tissue characterization and, possibly, the use of quantitative ultrasounds in the diagnostics and screening of malignant disease.

\section{Acknowledgements}

The authors wish to thank the State of Ohio for the funding that supported this project. We thank Dr. Stanislav Rokhlin for providing ultrasonic instruments and assistance in developing experimental protocols, Dr. Lugen Wang for helpful discussions on the continuum model, and Bin Xie for help on sample mounts. We are also grateful to Mrs. Mary Marin, Karen Williams and Mr. Shown Coontz, for the preparation of tissue sections.

\section{References}

[1] P.R. Srinivas, B.S. Kramer and S. Srivastava, Trends in biomarker research for cancer detection, The Lancet Oncology 2(11) (2001), 698-704.

[2] J.E. Roulston, Screening with tumor markers: critical issues, Molecular Biotechnology 20 (2002), 153-162.

[3] P. Anker, H. Mulcahy, X.Q. Chen and M. Stroun, Detection of circulating tumour DNA in the blood (plasma/serum) of cancer patients, Cancer And Metastasis Reviews 18(1) (1999), 65-73. 
[4] I. Botezatu, O. Serdyuk, G. Potapova, V. Shelepov, R. Alechina, Y. Molyaka, V. Anan'ev, I. Bazin, A. Garin, M. Narimanov, V. Knysh, H. Melkonyan, S. Umansky and A. Lichtenstein, Genetic analysis of DNA excreted in urine: A new approach for detecting specific genomic DNA sequences from cells dying in an organism, Clinical Chemistry 46(8) (2000), 1078-1084.

[5] M. Bloching, A. Hofmann, C. Lautenschlager, A. Berghaus and T. Grummt, Exfoliative cytology of normal buccal mucosa to predict the relative risk of cancer in the upper aerodigestive tract using the MN-assay, Oral Oncology 36(6) (2000), 550555.

[6] D.A. Ahlquist, J.E. Skoletsky, K.A. Boynton, J.J. Harrington, D.W. Mahoney, W.E. Pierceall, S.N. Thibodeau and A.P. Shuber, Colorectal cancer screening by detection of altered human DNA in stool: Feasibility of a multitarget assay panel, Gastroenterology 119(5) (2000), 1219-1227.

[7] Report of Active Disease Management Session of the National Institutes of Health's BECON 2002: Sensors for Biological Research and Medicine, Bethesda, Maryland (to appear), June $24-25,2002$

[8] M. Essenpreis, T.A. Desai, M. Ferrari and D.J. Hansford, Implantable Analyte Sensor, US Patent: 6,405,066 B1, June 11, 2002.

[9] S.J. Nass, C. Henderson and J.C. Lashof, eds, Chapter 2. Breast imaging and related technologies, in Mammography and Beyond, National Academy of Sciences, 2001.

[10] J. Ophir, I. Cespedes, H. Ponnekanti, Y. Yazdi and X. Li, Elastography - A Quantitative Method For Imaging The Elasticity Of Biological Tissues, Ultrasonic Imaging 13(2) (1991), 111-134.

[11] R. Muthupillai and R.L. Ehman, Magnetic resonance elastography, Nature Medicine 2(5) (1996), 601-603.

[12] T.A. Krouskop, T.M. Wheeler, F. Kallel, B.S. Garra and T. Hall, The elastic moduli of breast and prostate tissues under compression, Ultrasonic Imaging 20 (1998), 151-159.

[13] A.I. Lavrentyev and S.I. Rokhlin, Determination of elastic moduli, density, attenuation, and thickness of a layer using ul- trasonic spectroscopy at two angles, Journal of the Acoustical Society of the America 102(6) (1997), 3467-3477.

[14] Y.C. Fung, A First Course in Continuum Mechanics: for Physical and Biological Engineers and Scientists, Englewood Cliffs, Prentice Hall, NJ, 1994.

[15] T. Mura, Micromechanics of Defects in Solids, Kluwer Academic Publisher, 1982.

[16] G. Venkataraman, L.A. Feldkamp and V.C. Sahni, Dynamics of Perfect Crystals, Cambridge, MIT Press, 1975.

[17] A.R. Leach, Molecular Modeling: Principles and Applications, Addison Wesley Longman Ltd., 1996.

[18] M. Ferrari, V.T. Granik, A. Imam and J.C. Nadeau, eds, Advances in Doublet Mechanics, Springer, 1997.

[19] J.L. Rose, Ultrasonic Waves in Solid Media, Cambridge University Press, 1999.

[20] J. Liu and M. Ferrari, A discrete model for the high frequency elastic wave examination on biological tissue, Manuscript accepted by Computer Modeling in Engineering and Sciences, 2002.

[21] M. Ferrari, Nanomechanics, and Biomedical Nanomechanics: Eshelby's Inclusion and Inhomogeneity Problems at the Discrete/Continuum Interface, Biomedical Microdevices 2(4) (2000), 272-281.

[22] J. Sakamoto, PhD Thesis, The Ohio State University Biomedical Engineering Center, expected 2003.

[23] F. Martin and C. Grove, Microfabricated drug delivery systems: concepts to improve clinical benefit, Biomedical Microdevices 3(2) (2001), 97-107.

[24] J. Lewis and M. Ferrari, BioMEMS for drug delivery applications, Invited chapter for "Lab-on-a-chip: chemistry in miniaturized synthesis an analysis systems." 2002.

[25] S.P. Bao, B.D. Thrall and D.L. Miller, Transfection of a reporter plasmid into cultured cells by sonoporation in vitro, Ultrasound In Medicine And Biology 23(6) (1997), 953-959.

[26] A.J. Coleman and J.E. Saunders, A review of the physicalproperties and biological effects of the high amplitude acoustic fields used in extracorporeal lithotripsy, Ultrasonics 31(2) (1993), 75-89. 


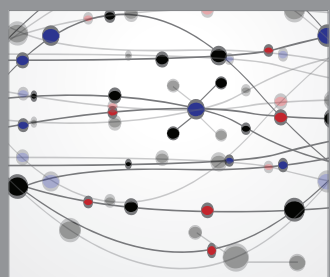

The Scientific World Journal
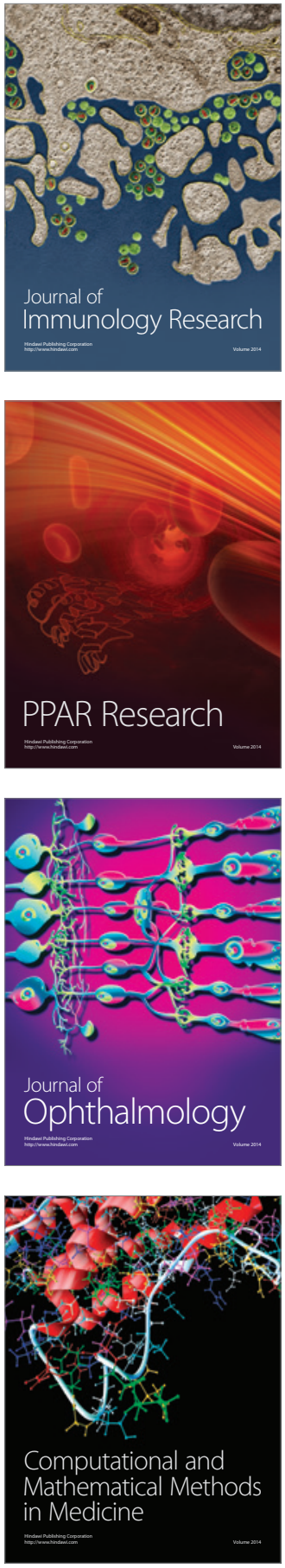

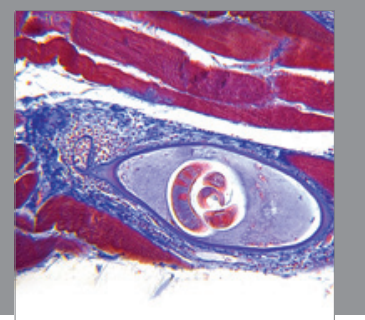

Gastroenterology

Research and Practice
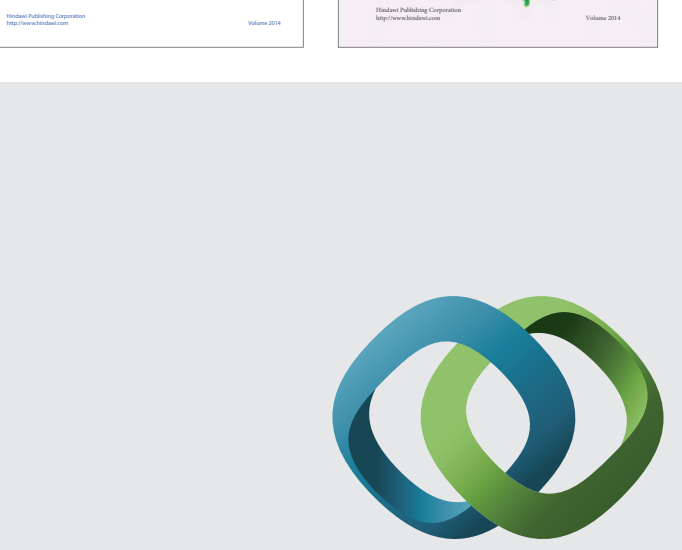

\section{Hindawi}

Submit your manuscripts at

http://www.hindawi.com
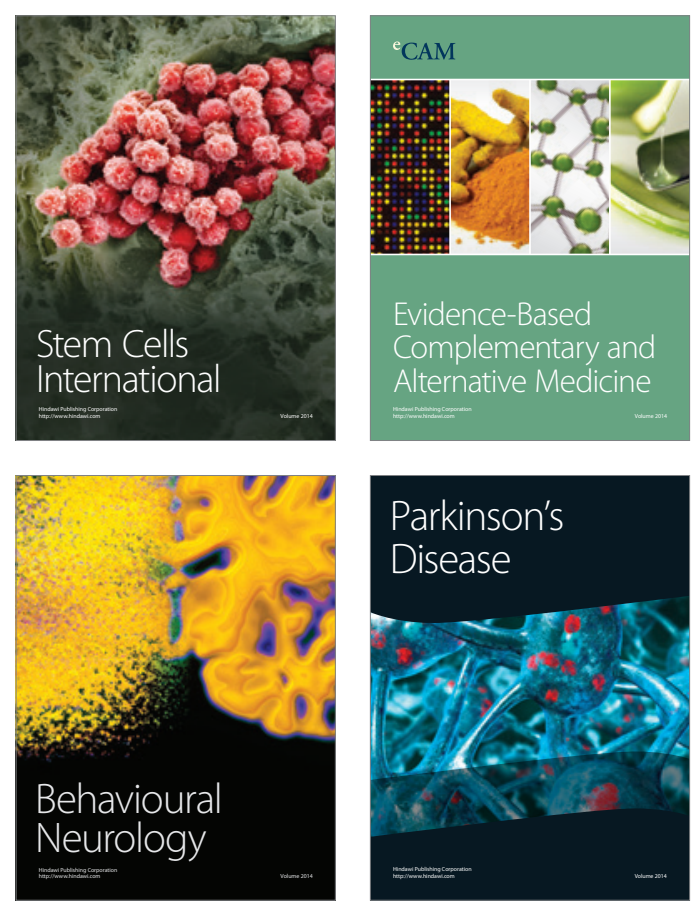

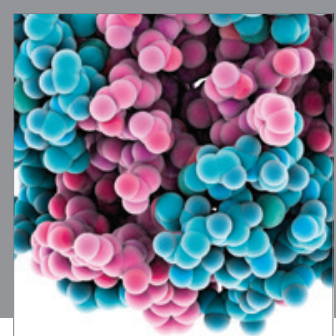

Journal of
Diabetes Research

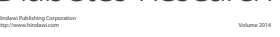

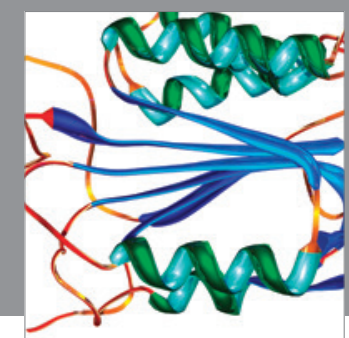

Disease Markers
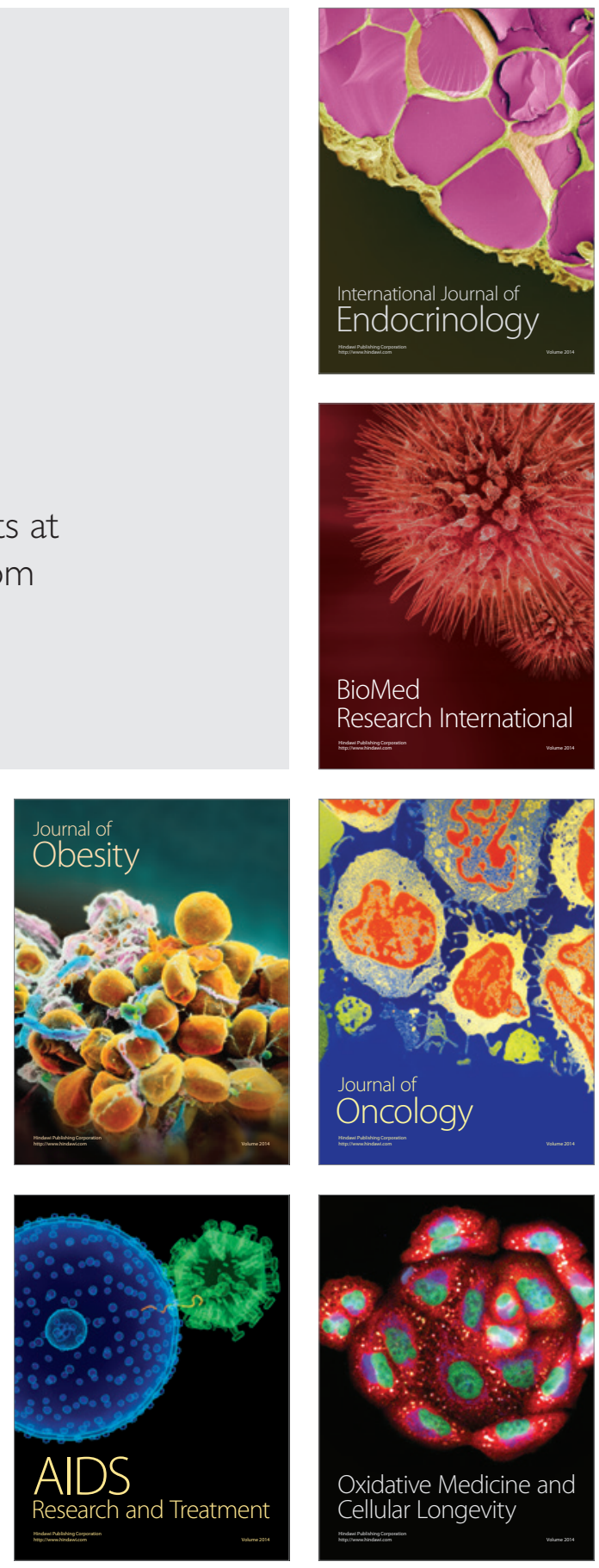